\title{
Optimization of Coal-fired Boiler on LS-SVM Model and PSO Algorithms
}

\author{
Yizhuo Zhang ${ }^{1, a^{*}}$, Hongjin Zhang ${ }^{2, b}$ and Weidong Zhang ${ }^{3, c}$ \\ Information Processing and Advanced Control Group, Shanghai Jiao Tong University, China. \\ aa82352851@163.com, bjindision@sjtu.edu.cn, cwdzhang@sjtu.edu.cn
}

Keywords: Boiler combustion optimization, LS-SVM, PSO algorithms, Nox emissions.

Abstract. With the deterioration of dust storm in recent years, environmental protection has become a topic for everyone. The most important problem of environmental protection comes from coal combustion which can be improved by combustion optimization. And combustion optimization has been proved to be an effective way to reduce the Nox emissions and improve boiler combustion efficiency by setting the operating parameters. The aim of this work is to achieve optimization of the coal-fired boiler by least square-support vector machine ( $L S-S V M)$ model and PSO algorithm. In this paper, $L S-S V M$ was applied to build Nox emissions model, carbon content of fly ash model and flue gas temperature model. Thereafter, based on the above models, we select PSO algorithm and GA to solve the problem. The results of the experiment demonstrate that $P S O$ algorithm is superior to $G A$ and it is effective on improving boiler's efficiency and reducing Nox emissions.

\section{Introduction}

Recently, environmental protection problem has become urgent problem. In China, coal-fired power accounted for three-quarters of the countries' electricity and $90 \%$ of the power comes from the coal combustion [1]. But most of the boilers are old-type which have low efficiency and high Nox emissions. Under the pressure of energy saving and shrinking margins, coal-fired power plants need to improve its combustion efficiency and reduce Nox emissions. Research has shown that selective catalytic reduction (SCR) technology is useful but a lot of cost [2].

Combustion optimization has been proved to be an effective way to reduce the Nox emissions [3] and unburned carbon in fly ash [4] by carefully setting the operational parameters. Ken Stuck Meyer [5] took Sioux Boiler Plant II (500MW) as the object and reduced Nox emissions. With the recommended input, Nox emissions were reduced by $25 \%$. Dharmarakan took Okanagan Boiler Plant I in Texas State (666MW) as the object, compared with the previous state, and reduced Nox emissions to $18 \%$, while ash content in the flue gas was reduced by $86 \%$ [6].

Due to the complexity of boiler combustion model, it is difficult to establish a precise mathematical model for it. However, artificial intelligence methods may be applied on alternative models [7]. Domestic common modeling methods are based on state estimation, fuzzy theory and artificial neural networks [8]. Long training time, the random result and over-learning problem are the shortcomings of these methods. In this paper, we use the method of $L S-S V M$ to model the temperature of flue gas, unburned carbon, Nox emissions and combustion efficiency respectively. Based on the models, $P S O$ algorithm and $G A$ are applied to optimize the operating parameters of the coal-fired boiler. The results of the experiment show that $P S O$ algorithm can improve combustion efficiency and reduce Nox emissions.

\section{Boiler Combustion Model}

In this paper, we take a tangentially boiler as the object which the maximum pressure can reach 600MW. In this study, we use $L S-S V M$ to model coal-fired boiler.

$\boldsymbol{L S}$-SVM. Recently, Support Vector Machines, as a new learning method, is applied to the modeling. Compared with neural networks, it will convert the problem into a quadratic optimization problem. Theoretically, it is able to reach global optimum, avoiding getting local optimum [9]. $L S S V M$ is an improved support vector machine method, using linear equations instead of convex 
quadratic programming $(Q P)$ problem [10]. It has strong generalization ability, short training time, and the accurate result that can be widely used [11].

We denote a bag as $\left\{x_{i}, y_{i}\right\}_{i=1}^{N}, x_{i} \in R^{n}$, in which $i$ is the bag index. And the label of bag is denoted as $y_{i} \in\{-1,1\}$. According to the Vapnik's original separation model [12] and avoiding the phenomenon that separating hyper plane does not exist, we introduce a slack variable $\zeta$ in Eq. 1:

$$
\begin{cases}y_{i}\left[\omega^{T} \phi\left(x_{i}\right)+b\right] \geq 1-\zeta_{i}, & i=1, \ldots, N, \\ \zeta_{i} \geq 0, & i=1, \ldots, N,\end{cases}
$$

According to the best principle to solve problems [13], quadratic problem can be converted to a linear problem about error $\omega$ and $e$ as Eq. 2 follows:

$$
\left[\begin{array}{cc}
0 & 1_{N}^{T} \\
1_{N} & \Omega+\gamma^{-1} I_{N}
\end{array}\right]\left[\begin{array}{l}
b \\
\alpha
\end{array}\right]=\left[\begin{array}{l}
0 \\
Y
\end{array}\right]
$$

Among them, $I_{N}$ is the unit matrix, $\Omega=\phi\left(x_{i}\right)^{T} \phi\left(x_{j}\right)=K\left(x_{i}, x_{j}\right) \in R^{N \times N}$ is the kernel function in Gaussian space. In this paper, the kernel function is selected as $K\left(x_{i}, x_{j}\right)=\exp \left(-\left\|x_{i}-x_{j}\right\|^{2} /\left(2 \sigma^{2}\right)\right)$.

The Structure and Sample of Model. However, we should build the structure of the model before using $L S-S V M$. In this paper, we randomly selected experimental data that are normalized with mapstd function for different active data between $200 \mathrm{~W}$ to $600 \mathrm{~W}$. We have a form of 100 sets of data, where 90 sets of data are training data, 10 sets of data are prediction data. Table 1 shows part of the data. We select twenty-one variables as inputs, three variables as output. The structure of model is shown in Fig. 1.

Table 1. Condition Working Data

\begin{tabular}{|c|c|c|c|c|}
\hline Data & Load & $\psi\left(\mathrm{O}_{2}\right)$ & $\ldots$ & Nox \\
\hline 1 & 505 & 3.6 & $\ldots$ & 21.6 \\
\hline 2 & 455 & 4.3 & $\ldots$ & 22.1 \\
\hline$\ldots$ & $\ldots$ & $\ldots$ & $\ldots$ & $\ldots$ \\
\hline 100 & 439 & 2.2 & $\ldots$ & 19.6 \\
\hline
\end{tabular}

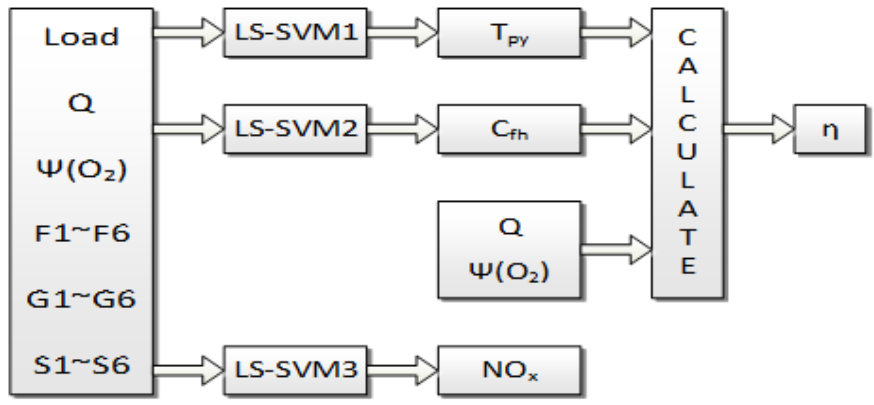

Fig. 1. The Structure of Model

In which, Load is the effectiveness of power, $Q$ is the total amount of fuel, $\psi\left(O_{2}\right)$ is the oxygen amount in boiler mouth, F1-F6 are first air ports, G1-G6 are the amount of coal ports and S1-S6 are secondary air ports. $L S-S V M$ method respectively is applied on flue gas temperature, unburned carbon and Nox emissions. We calculate the efficiency of the boiler with simplified formula by combining with the total amount of coal and oxygen. 
The Parameters and Simulation. $L S-S V M$ method has two parameters-kernel function $\sigma$ and regularization parameter $c$. We use a three-step search optimization techniques to determine these two parameters [14], calculated parameters through it are shown in Table 2, in which, $L S$-SVM1, $L S-S V M 2$ and $L S$-SVM3 represent the model of flue gas temperature, unburned carbon and Nox emissions.

Table 2. Parameters of Model

\begin{tabular}{|c|c|c|c|}
\hline Parameters & $L S$-SVM1 & LS-SVM1 & LS-SVM1 \\
\hline$\sigma$ & 4 & 0.4 & 1 \\
\hline$c$ & 100 & 60 & 2 \\
\hline
\end{tabular}

According to the principle of minimum training set and prediction error, Fig. 2 to Fig. 4 show the three models in simulation, where the blue line represents the actual values and the red line represents the predicted value.

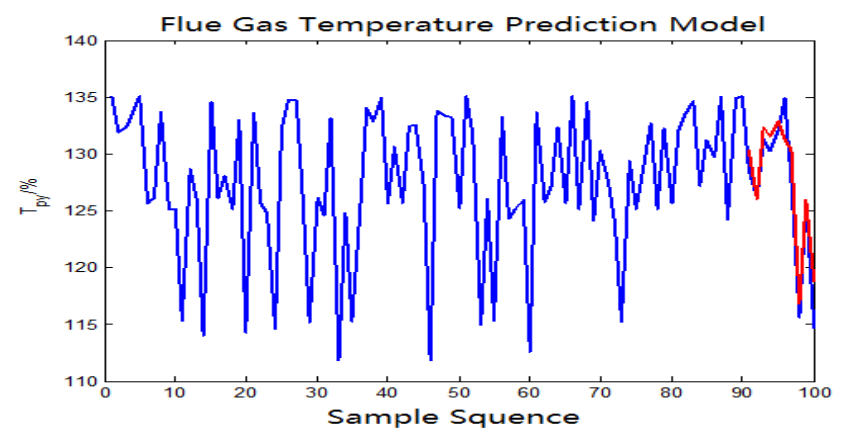

Fig. 2. Flue Gas Temperature Prediction Model

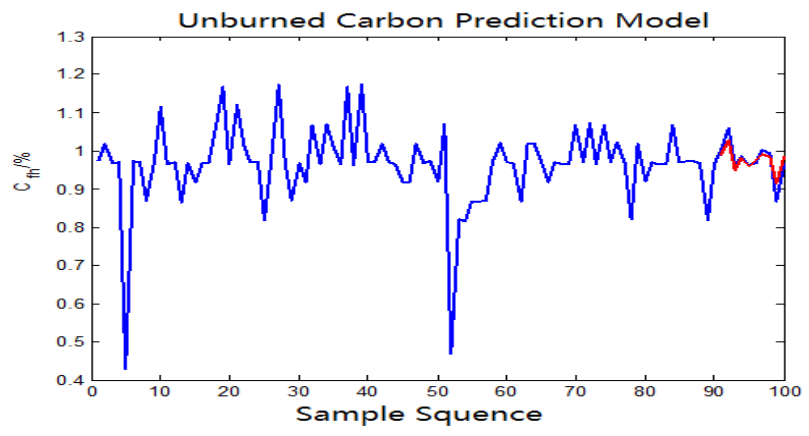

Fig. 3. Unburned Carbon Prediction Model

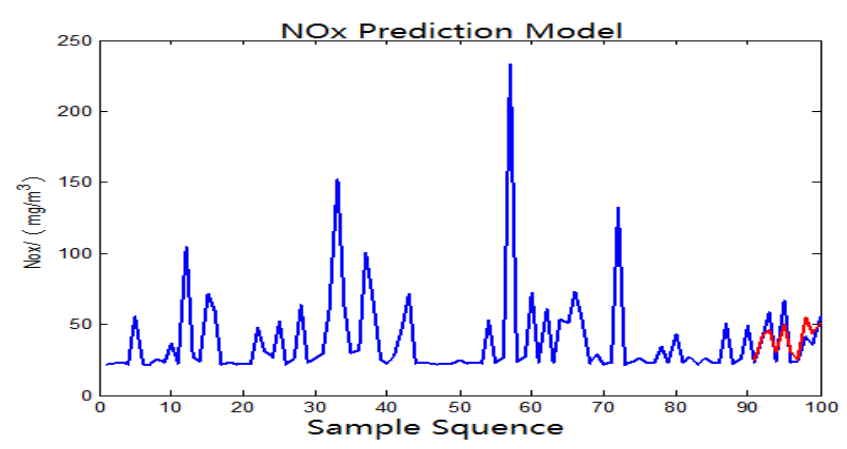

Fig. 4. Nox Prediction Model

Combustion Efficiency Prediction Model. There are two calculation standard of boiler combustion efficiency. One is Chinese test standard (GB PTC), the other is the American Society of Mechanical Engineers test standard (ASME PTC) [15]. In this paper, we choose positive balance 
method to calculate the combustion efficiency of the boiler. But the actual boiler combustion efficiency calculation is very complicated, so we use a simplified formula in Eq. 3:

$$
\eta=\frac{D}{D+\mu(3.5 \alpha+0.5) \times\left(T_{p y}-T_{a}\right) / 100}
$$

Using $L S-S V M$ to predict boiler combustion efficiency, Fig. 5 shows the results of the simulation:

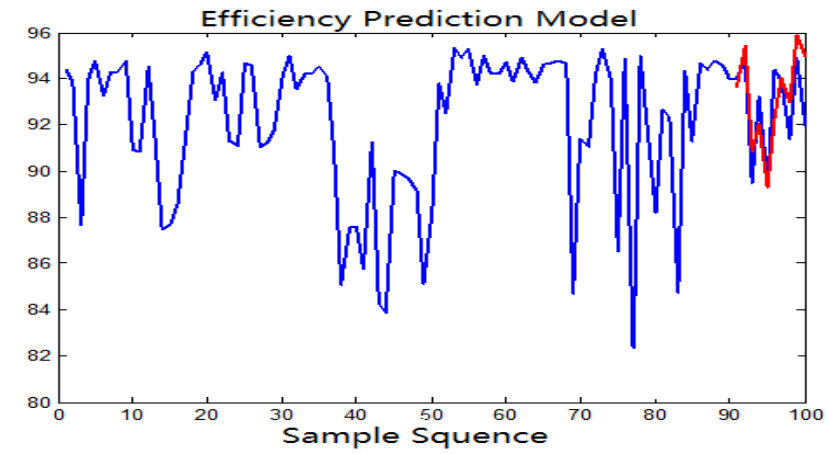

Fig. 5. Efficiency Prediction Model

\section{PSO Algorithm and Optimization}

Principle. We denote a set as $z_{i}=\left(z_{i 1}, z_{i 2}, \cdots, z_{i D}\right)$ that represents particle's position, a set as $v_{i}=\left(v_{i 1}, v_{i 2}, \cdots, v_{i D}\right)$ that represents particle's velocity, a set as $p_{i}=\left(p_{i 1}, p_{i 2}, \cdots, p_{i D}\right)$ that represents best position of $i$ particle and a set as $p_{g}=\left(p_{g 1}, p_{g 2}, \cdots, p_{g D}\right)$ that represents best position of all particles. The particles update their speed and location each iteration according to Eq. 4 as follows:

$$
\left\{\begin{array}{l}
v_{i d}^{k+1}=\omega v_{i d}^{k}+c_{1} r_{1}\left(p_{i d}-z_{i d}^{k}\right)+c_{2} r_{2}\left(p_{g d}-z_{i d}^{k}\right) \\
z_{i d}^{k+1}=z_{i d}^{k}+v_{i d}^{k+1}
\end{array}\right.
$$

In which, $k$ represents the number of iterations, $c_{1}$ and $c_{2}$ represent learning factor, $r_{1}$ and $r_{2}$ are random from $[0,1], \omega$ represents inertia factor.

Selection Parameters. PSO has a great advantage over the other optimization algorithms, but the choice of parameters is difficult. After a lot of experiments, it proves its parameters without complexity select [16], and even some parameters can be removed, the most authoritative is the space graphical methods. Written in Eq. 5 matrix:

$$
\left[\begin{array}{l}
x_{k+1} \\
v_{k+1}
\end{array}\right]=\left[\begin{array}{cc}
1-c & \omega \\
-c & \omega
\end{array}\right]\left[\begin{array}{l}
x_{k} \\
v_{k}
\end{array}\right]+\left[\begin{array}{l}
c \\
c
\end{array}\right] p
$$

According to the principle of dynamic system stability, range of parameters can be solved as Eq. 6:

$\omega<1, \quad c>0, \quad 2 \omega-c+2>0$

(6)

Optimization Process. In this paper, Nox emissions and the combustion efficiency are goals. You can get the best value of the operating parameters by PSO algorithm, and then fed them back to the control systems to achieve combustion optimization goals, which diagram is Fig. 6: 


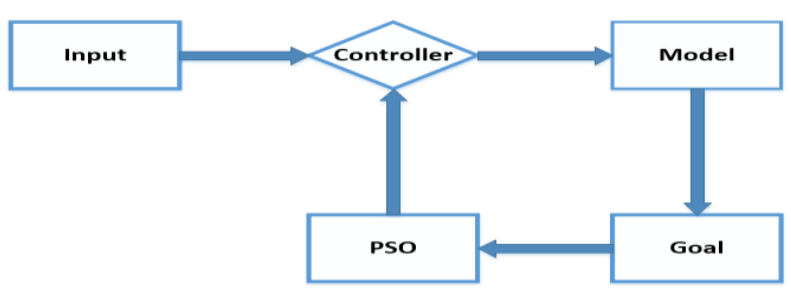

Fig. 6. Optimization Process

Simulation. Applying PSO on combustion efficiency and Nox emissions, the experiment on matlab shows in Fig. 7 and Fig. 8:

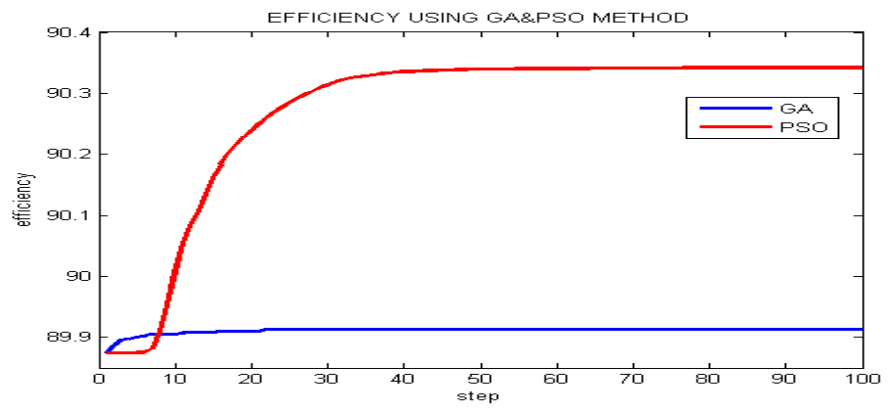

Fig. 7. Optimization of Efficiency

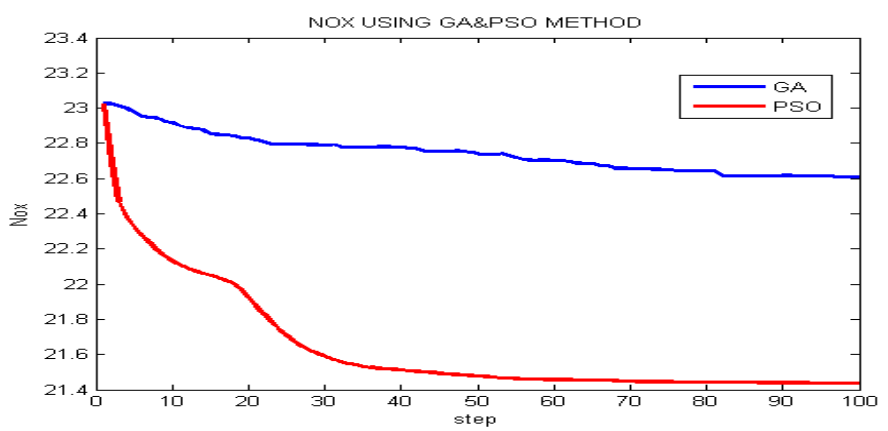

Fig. 8. Optimization of Nox

Through the above diagrams, and specific datas are in Table 3:

Table 3. Compare parameters

\begin{tabular}{|c|c|c|}
\hline Optimum & Nox & Efficiency \\
\hline$G A$ & 22.7 & 89.9 \\
\hline PSO & 21.4 & 90.35 \\
\hline
\end{tabular}

Above mentioned can be seen, $P S O$ algorithm not only has shorter time but also has more quickly convergence rate. Moreover, $P S O$ algorithm is superior to GA algorithm and it is better to improve combustion efficiency and reduce Nox emissions.

\section{Conclusions}

This paper use the $L S-S V M$ and PSO algorithm to optimize coal-fired boiler. Based on the $L S-S V M$, we establish the models of unburned carbon, exhaust gas temperature and Nox emissions. Applying $P S O$ algorithm to the model of coal-fired boiler, we achieve the lower Nox emissions and higher combustion efficiency. This paper compares $P S O$ algorithm with $G A$. Comparative results show that PSO algorithm can reduce Nox emissions and improve boiler combustion efficiency, with short 
optimized time and good optimization results. It shows that the application of swarm intelligence algorithms can solve complex industrial problems. This combustion optimization control method can achieve high efficiency and low Nox emissions.

\section{References}

[1] Hong, Jongsup. Analysis of oxy-fuel combustion power cycle utilizing a pressurized coal combustor. Energy 34.9 (2009): 1332-1340.

[2] Blakeman, Philip G., et al. Developments in diesel emission aftertreatment technology. No. 2003-01-3753. SAE Technical Paper, 2003.

[3] Zheng, Ligang, et al. Combining support vector regression and ant colony optimization to reduce NOx emissions in coal-fired utility boilers. Energy \& Fuels 22.2 (2008): 1034-1040.

[4] CHEN, Min-sheng, and Ding-ping LIU. Optimized Control of Carbon Content in Utility Boilers' Fly Ash [J]. Power Engineering 4 (2005): 021.

[5] Cremer, Marc, et al. Sub 0.15 lb/MBtu NOx Emissions Achieved with ALTA on a 500 MW Cyclone-Fired Boiler. Proceedings of the 31st International Technical Conference on Coal Utilization \& Fuel Systems, Clearwater, FL. 2006.

[6] Frost, G. J., et al. Effects of changing power plant NOx emissions on ozone in the eastern United States: Proof of concept. Journal of Geophysical Research: Atmospheres (1984-2012) 111.D12 (2006).

[7] $\mathrm{Wu}$, Feng, et al. A comparative study of the multi-objective optimization algorithms for coal-fired boilers. Expert Systems with Applications 38.6 (2011): 7179-7185.

[8] Gu, Yan-Ping, Wen-Jie Zhao, and Zhan-Song Wu. Combustion optimization for utility boiler based on least square-support vector machine. Zhongguo Dianji Gongcheng Xuebao(Proceedings of the Chinese Society of Electrical Engineering). Vol. 30. No. 17. Chinese Society for Electrical Engineering, 2010.

[9] KONG, Liang, et al. Adaptive Kriging model for NO_x emissions from utility boilers [J]. Journal of Tsinghua University (Science and Technology) 5 (2008): 021.

[10] Yan, Weiwu, Huihe Shao, and Xiaofan Wang. Soft sensing modeling based on support vector machine and Bayesian model selection. Computers \& Chemical Engineering 28.8 (2004): 1489-1498.

[11]Suykens, J. A. K., and Joos Vandewalle. Recurrent least squares support vector machines. Circuits and Systems I: Fundamental Theory and Applications, IEEE Transactions on 47.7 (2000): 1109-1114.

[12] Vapnik, Vladimir. The nature of statistical learning theory. Springer Science \& Business Media, 2013.

[13] MacKay, David JC. Bayesian interpolation. Neural computation 4.3 (1992): 415-447.

[14] Guo, Hui, H. P. Liu, and Ling Wang. Method for selecting parameters of least squares support vector machines and application. Journal of System Simulation 18.7 (2006): 2033-2036.

[15]Jun, Xiang, et al. An experimental research on boiler combustion performance. Fuel Processing Technology 68.2 (2000): 139-151.

[16] Trelea, Ioan Cristian. The particle swarm optimization algorithm: convergence analysis and parameter selection. Information processing letters 85.6 (2003): 317-325. 